\title{
GMM based Image Segmentation and Analysis of Image Restoration Tecniques
}

\author{
Shilpa Hatwar \\ ME Student: E and TC Dept \\ Dr. D.Y.P.S.O.E. \\ Pune, India
}

\author{
Anil Wanare \\ Professor: E and TC Dept \\ B.D.C.O.E. \\ Pune, India
}

\begin{abstract}
Now days cauterization of images and video is easy with the advanced technologies in camera. But this images and videos are get easily contaminated by noise due to the characteristics of the image sensors due to this they are mostly blurred so we can loss important data. To avoid this problem we proposed an algorithm for segmentation based on Gaussian mixture model (GMM) and restoration technique with spatial smoothness constraints. The researchers worked on single type of image but the different environmental images are may be affected due to different noises so that researched work is not suitable for all environmental conditions. The proposed algorithm is works on the all type of images, which can remove noises from different diverse field of images with calculating different image parameter. From all of this we can get the optimum solution of suitable filters for combination of image and noise for reduction of noise by comparing of all of that. Here we also present the algorithm for video segmentation \& restoration.
\end{abstract}

\section{General Terms}

Segmentation algorithm, image parameters, image restoration techniques.

\section{Keywords}

GMM segmentation algorithm, spatial domain filter, PSNR, MSE, transform domain filter.

\section{INTRODUCTION}

Now days capturing of Images video with advanced cameras is sophisticated. However, it typically contains some degree of noise and blurriness. In low-light situations, due to camera moving can disturb an image. If the exposure time reduced to remove blur due to motion in the scene, intensity and noise may be increased beyond acceptable levels. The act of restoring an image to remove noise and blur is typically an under-constrained problem. Information lost during an observation process needs to be restored with prior information about original images to achieve real visualization. Image segmentation is another major problem in image processing and computer vision. Image segmentation is the process of separating or grouping an image into different parts. These parts normally correspond to something that humans can easily separate and view as individual objects. Computers have no means of intelligently recognizing objects, and so many different histograms, or information about the pixels that indicate edges or boundaries or texture information. When a process of classification approaches the problem of image segmentation, several methods exist. Finite mixture models have attracted considerable interest for image segmentation. However, the application of finite mixtures model to image segmentation presents some difficulties. For the classical mixture statistical, each pixel must be associated with exactly one class. This assumption may be not realistic.
Thus, several methods have been proposed to circumvent this problem. This approach provide satisfactory results in many case, but most case the assumption of a single Gaussian distribution typically limits image segmentation accuracy. For the process of image segmentation and restoration algorithm developed based on Gaussian mixture model, which based on a parametric model in which the probability density function of the gray levels in the image is a mixture of Gaussian density functions. This model has received considerable attention in the development of segmentation and restoration algorithms and it is applicable for all types of image i.e. the natural image, underwater image, biomedical image and it improves the image quality by calculating different quality parameters of image. Our main work is to process on both static and dynamic images from different diverse field of images

\section{LITERATURE SURVEY}

The various algorithms for images and video segmentation based on background and foreground gives by Heikkila and Olli, Pfinder, $w^{4}$ Lots, Halvey, cutler, and wallflower [1]. From this method, a few methods are expensive and others, which are not expensive, are not giving reliable segmentation. The various methods proposed by researchers for segmentation process [2] K. Satish gives a concept about Automatic Image Segmentation using Wavelets [3]. Matthew Marsh defines a various different segmentation techniques [4]. Christopher's Nikou [5] give a new concept on A new Bayesian model for image segmentation Gaussian mixture models (GMM) with spatial smoothness constraints. Mohand Saïd Allili [9] gives the concept segmentation with Wavelet Modeling Using Finite Mixtures of Generalized Gaussian Distributions. An EM algorithm developed to estimate parameters of the Gaussian mixtures model. The research works on Image restoration as Anil L Wanare [6] gives Optimum solution for Linear Restoration in the Spatial Domain. Formatting gradient [7] distributions Gaussian mixture modeling and efficient Gaussian mixture model gives the concept of GMM model. S.-J. Ko and S.-J. Lee and D. Brownrig gives the concept of restoring of image of weighted median filter [10][11]. The concept of median filter is also explained by T. Sun and Y. Neuvo and D. Florencio and R. Schafer [12][13].How-Lung Eng explain the switching median filter for the restoring of images[14]. However, the no one was work on different images of diverse field of images. Here we proposed the method, which is work for segmentation of video, and Images on GMM (Gaussian Mixture Model) based method and restoring of video as well as different diverse field images with spatial domain filters like median filter, mean filter and wiener filter and one wavelet transformation filter based on GMM. 


\section{PROPOSED METHODOLOGY}

Our proposed system based on Gaussian mixture model for segmentation and restoration of dynamic and static image. Segmentation is the process of dividing an image into different types of regions or classes, recognizing of objects etc by machine. All of these can do after segmentation of image. Restoration of image i.e. removing of noise can do after segmentation process. We use here static and dynamic image. Static images are of 5 types i.e. biomedical image, underwater image, natural image, aerial image, texture image this images may be have different noises such as salt \& pepper noise, Gaussian noise, speckle noise and Poisson noise. Here the noise is assumed to be additive and its mean is zero. The images with noise is can be expressed in following equation

$I=P+N, E(N)=0$

$I$ - image with noise.

$P$ - Original image.

$N$ - Added noise whose mathematical expectation is zero.

$$
\begin{aligned}
E(I) & =E(P+N) \\
= & E(P)+E(N) \\
& =E(P)
\end{aligned}
$$

\subsection{Implementation of segmentation and Restoration algorithm}

The segmentation algorithm based on a GMM algorithm. In addition, for the restoration process we use total four filters, three of spatial domain name as Mean filter, median filter and wiener filter. Moreover, one from transform Domain is wavelet filter based GMM. The flowchart of overall concept of proposed methodology is in fig. 1

\subsubsection{Apply input images}

First we have to be apply the video or frames as a input which may be noisy or noiseless

\subsection{2 . Setting up Gaussian parameters}

The number of file formats used in multimedia continues to proliferate. The length, width, frames per second, file format Number of frames is noted. The frames of the videos analyzed. The Gaussian parameters required for image are mean $\mu \mathrm{i}$ standard deviation $\sigma \mathrm{i}$, weight $\omega \mathrm{i}$ of each model are pre declared to $\mu$ INIT, $\sigma$ INIT, $\omega$ i for $1 / \mathrm{G}$.(i=1, $2, \ldots \mathrm{G})$. The value of the $\mu$ INIT is significantly disturbs primer sequence of the process. The value of $\sigma$ INIT is directly proportional to the updating rate. That is why $\sigma$ should be selected as a moderate value in for better background and foreground separation.

\subsection{Segmentation Algorithm}

The segmentation algorithm is shows in Fig. 2

\subsubsection{Matching Assessment}

There will be the Threshold for light variations like the objects, which becomes static after a particular time. Shadow, and light illumination determined by experiments and a suitable value had chosen due to this de-noising effect does not get degraded.

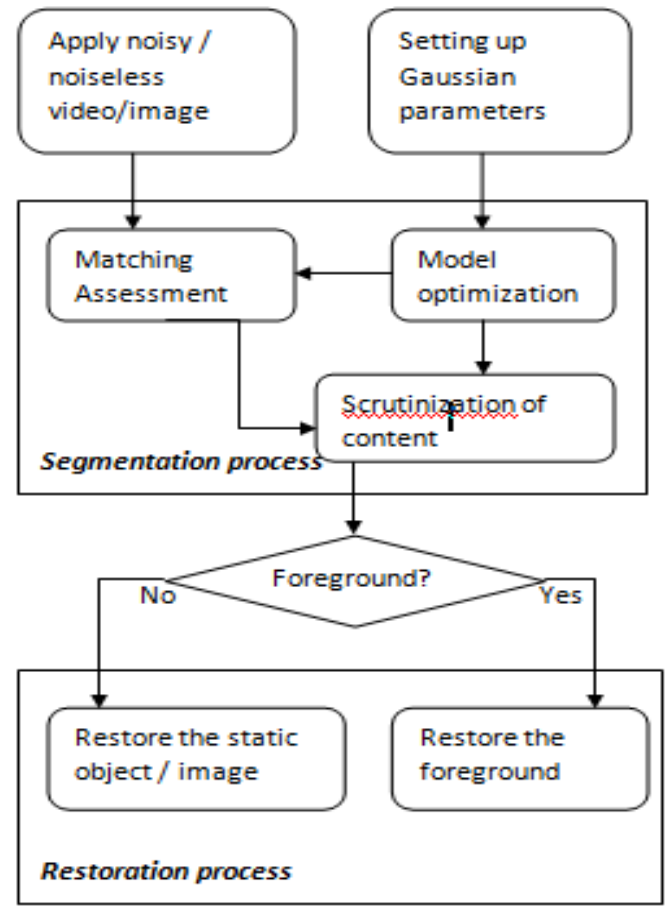

Fig.1 flow chart of the proposed concept

\subsubsection{Matching Reconfiguration:}

From the frame, each pixel value Xt compared with the prevailing $\mathrm{K}$ Gaussian distributions to differentiate the matched and unmatched pixel. This is important concept when different regions have different lighting. Three cases are undertaking in model updating process. If the absolute value of the change in current pixel value with the mean is less than sequence the twice of variance then those pixel values corresponds to background and then the weights are sorted in descending order. Therefore, the result, the least probable distribution will be at the bottom and the highest weight at the Sequence top.

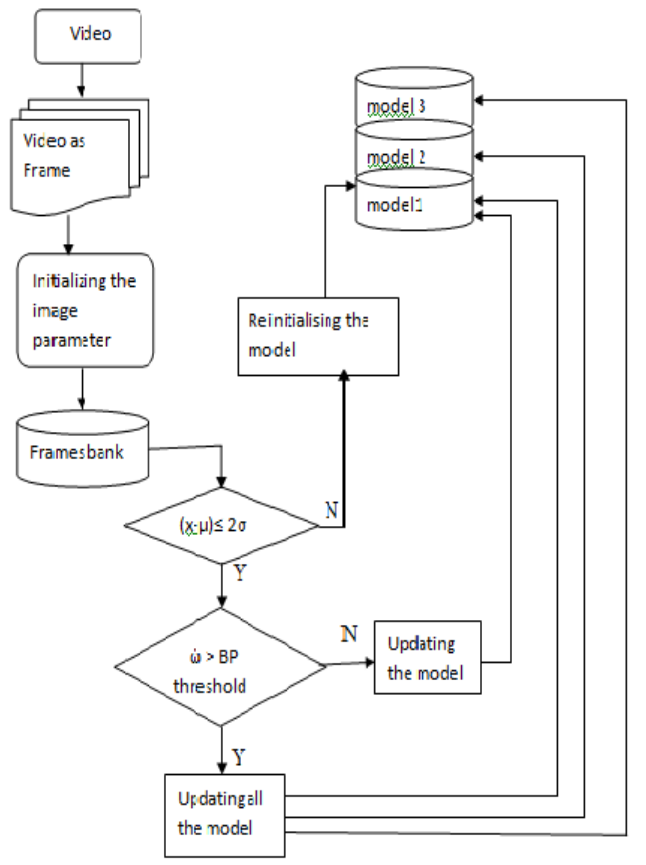

Fig. 2 GMM based segmentation algorithm 


$$
\text { Diff }=\text { threshold } * \text { sigma }
$$

Where, Diff is the absolute value of difference between current frame pixel value and mean of $\mathrm{G}$ model. Further to the execution of the equation the rate at which the reconfiguring matching values is determined and weight variance and sigma are being changed accordingly. This change follows on three different classifications. On matching weights, variance, and mean being modified, for least weighed model. On unmatching the variance is restored with initial value, weight is multiplied with a factor of 0.5 and mean is changed with pixel of current frame. On a special case where matching value exceeding background proportion needs model optimization but at phased manner in order to do that weight, variance, and mean being modify for all three GMM models. The parameters of the distribution are up- dated as shown in Equation (4-6). The update factor varies according to the, matching judgment process.

$$
\begin{aligned}
& \mu=(1-\rho) \mu+\rho x \\
& \sigma=\sqrt{1-\rho \sigma^{2}+\rho(x-\text { ? })^{2}}
\end{aligned}
$$

The update factor varies according to the, matching judgment process. The update equations for the slight and deep variations cases are as follows:

$$
\begin{aligned}
& \rho=e^{\frac{(x-\mu)^{2 \sigma}}{(-2 \sigma 2)(2 \pi \sigma)}} \\
& \rho=\beta^{*} \frac{\alpha}{\sigma}
\end{aligned}
$$

Where, $\rho$ is directly proportional to $\alpha$ and it has a major impact in the update speed of $\mu-\sigma$. As the weight changes, $\rho$ may become very large, even larger than one, so it is necessary to set an upper limitation for $\rho$. One of the significant advantages of this method is that when something allowed becoming part of the background, it does not destroy the existing model of the background. Therefore, if an object is stationary just long enough to become part of the background and then it moves, the distribution describing the previous background still exists with the same $\mu$ and $\sigma$, but a lower $\omega$ and will be quickly re-incorporated into the background.

\subsubsection{Scrutinization of Content:}

Based on the matching assessment, silhouette image generated. Background proportion value help us in assisting the pixel whether it belongs to foreground and slighter variation in background. Background proportion threshold should be a trade-off value, on selection of higher value leads to delayed optimization of background model and lower value will fail to identify foreground.

\subsection{Restoration Process}

Restoration process of model is as follows

Based on the silhouette frame de-noising is applied. In that frame, largely 60 to 70 background and rest will occupy percentage of the frame by foreground. Here we apply same filter for foreground and background. We apply first median filter [14], mean filter [20], wiener filter [19], and wavelet filter based on GMM [18]. After separating, the frames of foreground and Background apply this filter to each combination of image and noise.

\section{RESULT AND EXPERIMENT}

This section presents the results obtained from the experimentation on the proposed GMM segmentation and restoration algorithm by applying spatial domain filters and transform domain filter. Here Barclay's standard images are used. Initially the process is apply on the static images for the comparative analyses on the base of various parameters which gives a suitable optimal solution for the selection of filter with respect combination of noise and types of image. The proposed system is applied to all combination of images i.e. medical images, natural images, aerial images and texture images with noises like salt \& pepper, Gaussian noise, speckle noise and Poisson noise to get a solution for which combination of noise and image we should apply the filter from spatial domain or from transform domains wavelet based GMM. Here shows the output for the medical images with different noises by applying different filters.

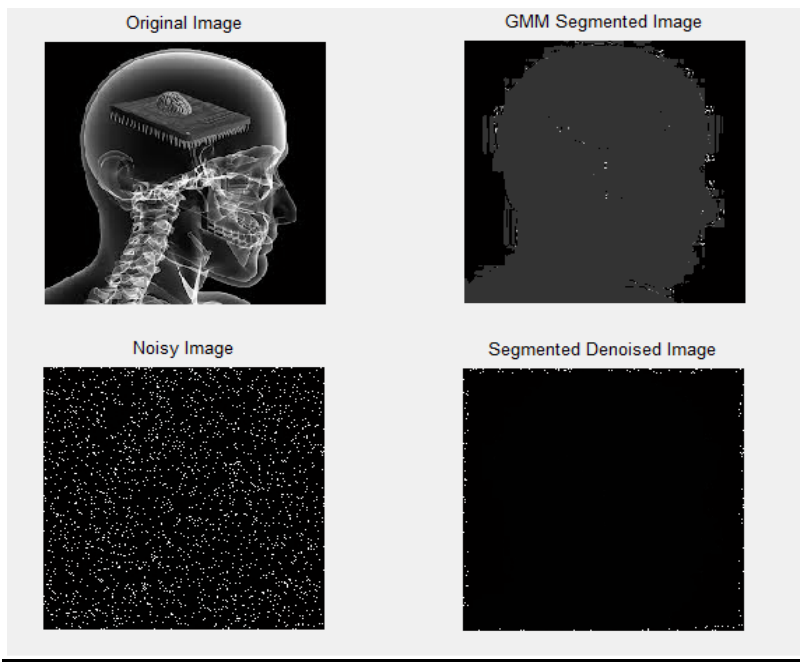

Fig. 3 GMM Segmentation and restoration process on medical image with salt and Pepper noise with mean filter.

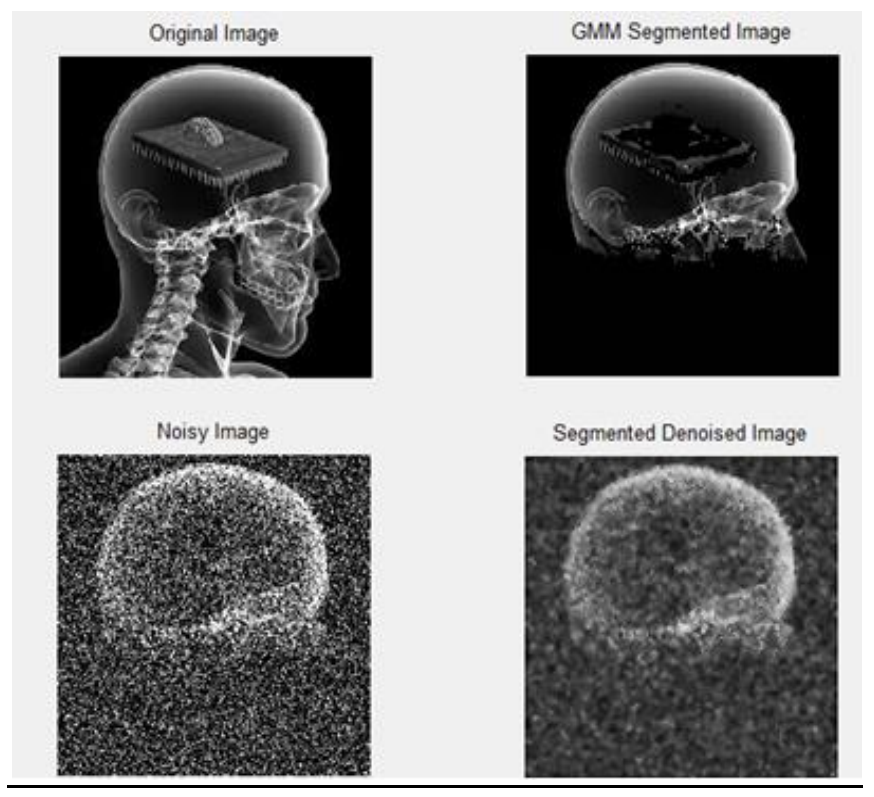

Fig. 4 GMM Segmentation and restoration process on medical image with Gaussian noise with mean filter 

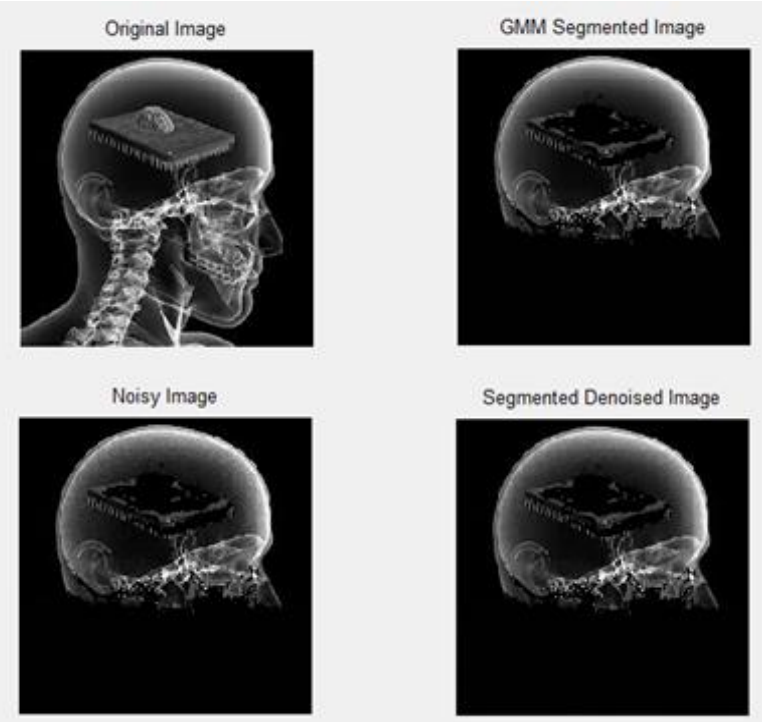

Fig. 5 GMM Segmentation and restoration process on medical image with Poisson noise with mean filter
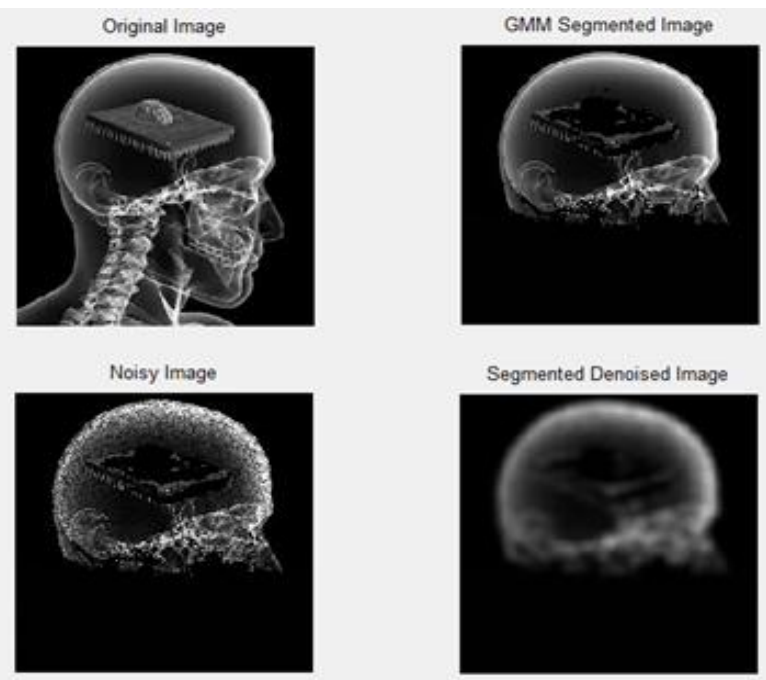

Segmented Denoised Image

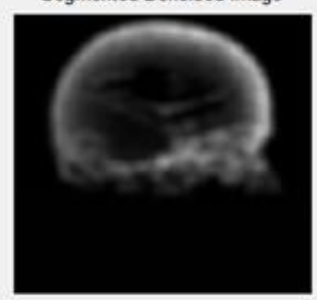

Fig. 6 GMM Segmentation and restoration process on medical image with speckle noise with mean filter

Table I Comparison between filters for different combination of noise and images

\begin{tabular}{|c|c|c|c|c|}
\hline \multirow{2}{*}{ Database } & \multirow{2}{*}{ Noise } & \multirow{2}{*}{$\begin{array}{l}\text { Applied } \\
\text { filters }\end{array}$} & \multicolumn{2}{|c|}{$\begin{array}{c}\text { Performance } \\
\text { parameter }\end{array}$} \\
\hline & & & $\begin{array}{l}P S N R(d \\
b)\end{array}$ & $\begin{array}{c}M S E \\
(d b)\end{array}$ \\
\hline \multirow{6}{*}{$\begin{array}{l}\text { Medical } \\
\text { image }\end{array}$} & $\begin{array}{c}\text { Salt \& Pepper } \\
\text { Noise }\end{array}$ & \multirow{4}{*}{$\begin{array}{l}\text { Mean } \\
\text { filter }\end{array}$} & 18.4229 & 934.95 \\
\hline & $\begin{array}{c}\text { Gaussian } \\
\text { noise }\end{array}$ & & 27.9243 & 104.87 \\
\hline & Poisson noise & & 36.8207 & 13.52 \\
\hline & Speckle noise & & 31.8439 & 42.52 \\
\hline & $\begin{array}{c}\text { Salt \& Pepper } \\
\text { Noise }\end{array}$ & \multirow{2}{*}{$\begin{array}{l}\text { Median } \\
\text { filter }\end{array}$} & 27.4036 & 118.22 \\
\hline & $\begin{array}{c}\text { Gaussian } \\
\text { noise }\end{array}$ & & 28.5022 & 28.50 \\
\hline
\end{tabular}

\begin{tabular}{|c|c|c|c|c|}
\hline & Poisson noise & & 37.0989 & 12.68 \\
\hline & Speckle noise & & 28.6428 & 88.87 \\
\hline & $\begin{array}{c}\text { Salt \& Pepper } \\
\text { Noise }\end{array}$ & & $\begin{array}{c}14.2652 \\
7\end{array}$ & 2435.3 \\
\hline & $\begin{array}{l}\text { Gaussian } \\
\text { noise }\end{array}$ & $\begin{array}{l}\text { Wiener } \\
\text { filter }\end{array}$ & 24.7056 & 220.04 \\
\hline & Poisson noise & & 25.7995 & 171.05 \\
\hline & Speckle noise & & 32.3428 & 38.78 \\
\hline & $\begin{array}{c}\text { Salt \& Pepper } \\
\text { Noise }\end{array}$ & & 21.2513 & 487.4 \\
\hline & $\begin{array}{l}\text { Gaussian } \\
\text { noise }\end{array}$ & $\begin{array}{c}\text { based } \\
\text { wavelet }\end{array}$ & 28.703 & 87.63 \\
\hline & Poisson noise & Filter & 33.169 & 31.33 \\
\hline & Speckle noise & & 25.719 & 174.3 \\
\hline & $\begin{array}{l}\text { Salt \& Pepper } \\
\text { Noise }\end{array}$ & Mean & 18.8159 & 854.0 \\
\hline $\begin{array}{l}\text { Natural } \\
\text { image }\end{array}$ & $\begin{array}{l}\text { Gaussian } \\
\text { noise }\end{array}$ & filter & 26.712 & 130.93 \\
\hline & Poisson noise & & 30.674 & 55.6751 \\
\hline & Speckle noise & & 26.960 & 130.923 \\
\hline & $\begin{array}{c}\text { Salt \& Pepper } \\
\text { Noise }\end{array}$ & & 25.8275 & 169.953 \\
\hline & $\begin{array}{l}\text { Gaussian } \\
\text { noise }\end{array}$ & $\begin{array}{l}\text { Median } \\
\text { filter }\end{array}$ & 26.1422 & 158.075 \\
\hline & Poisson noise & & 30.8532 & 53.4275 \\
\hline & Speckle noise & & 24.8221 & 214.225 \\
\hline & $\begin{array}{c}\text { Salt \& Pepper } \\
\text { Noise }\end{array}$ & & 14.143 & 2504.7 \\
\hline & $\begin{array}{l}\text { Gaussian } \\
\text { noise }\end{array}$ & $\begin{array}{l}\text { Wiener } \\
\text { filter }\end{array}$ & 20.530 & 575.51 \\
\hline & Poisson noise & & 20.648 & 560.02 \\
\hline & Speckle noise & & 20.473 & 583.11 \\
\hline & $\begin{array}{c}\text { Salt \& Pepper } \\
\text { Noise }\end{array}$ & & 20.053 & 642.27 \\
\hline & $\begin{array}{l}\text { Gaussian } \\
\text { noise }\end{array}$ & $\begin{array}{c}\text { based } \\
\text { wavelet }\end{array}$ & 27.513 & 115.26 \\
\hline & Poisson noise & Filter & 24.857 & 212.50 \\
\hline & Speckle noise & & 23.776 & 272.51 \\
\hline & $\begin{array}{l}\text { Salt \& Pepper } \\
\text { Noise }\end{array}$ & & 19.176 & 786.09 \\
\hline & $\begin{array}{l}\text { Gaussian } \\
\text { noise }\end{array}$ & Mean & 23.344 & 301.02 \\
\hline & Poisson noise & & 23.347 & 300.80 \\
\hline image & Speckle noise & & 22.625 & 355.24 \\
\hline & $\begin{array}{c}\text { Salt \& Pepper } \\
\text { Noise }\end{array}$ & $\begin{array}{l}\text { Median } \\
\text { filter }\end{array}$ & 21.903 & 419.53 \\
\hline
\end{tabular}




\begin{tabular}{|c|c|c|c|c|}
\hline & $\begin{array}{c}\text { Gaussian } \\
\text { noise }\end{array}$ & & 22.017 & 408.62 \\
\hline & Poisson noise & & 23.371 & 299.20 \\
\hline & Speckle noise & & 21.823 & 427.26 \\
\hline & $\begin{array}{l}\text { Salt \& Pepper } \\
\text { Noise }\end{array}$ & \multirow{4}{*}{$\begin{array}{l}\text { Wiener } \\
\text { filter }\end{array}$} & 15.040 & 2037.1 \\
\hline & $\begin{array}{c}\text { Gaussian } \\
\text { noise }\end{array}$ & & 19.330 & 758.62 \\
\hline & Poisson noise & & 19.658 & 703.46 \\
\hline & Speckle noise & & 19.402 & 746.18 \\
\hline & $\begin{array}{c}\text { Salt \& Pepper } \\
\text { Noise }\end{array}$ & \multirow{4}{*}{$\begin{array}{c}\text { GMM } \\
\text { based } \\
\text { wavelet } \\
\text { Filter }\end{array}$} & 20.174 & 624.63 \\
\hline & $\begin{array}{l}\text { Gaussian } \\
\text { noise }\end{array}$ & & 21.228 & 490.06 \\
\hline & Poisson noise & & 21.365 & 474.78 \\
\hline & Speckle noise & & 21.245 & 488.17 \\
\hline \multirow{16}{*}{$\begin{array}{l}\text { Texture } \\
\text { image }\end{array}$} & $\begin{array}{c}\text { Salt \& Pepper } \\
\text { Noise }\end{array}$ & \multirow{4}{*}{$\begin{array}{l}\text { Mean } \\
\text { filter }\end{array}$} & 20.501 & 579.40 \\
\hline & $\begin{array}{c}\text { Gaussian } \\
\text { noise }\end{array}$ & & 26.815 & 134.74 \\
\hline & Poisson noise & & 29.764 & 68.645 \\
\hline & Speckle noise & & 27.134 & 125.78 \\
\hline & $\begin{array}{l}\text { Salt\&Pepper } \\
\text { Noise }\end{array}$ & \multirow{4}{*}{$\begin{array}{l}\text { Median } \\
\text { filter }\end{array}$} & 25.418 & 186.74 \\
\hline & $\begin{array}{c}\text { Gaussian } \\
\text { noise }\end{array}$ & & 26.476 & 146.37 \\
\hline & Poisson noise & & 31.266 & 48.573 \\
\hline & Speckle noise & & 25.061 & 202.75 \\
\hline & $\begin{array}{c}\text { Salt \& Pepper } \\
\text { Noise }\end{array}$ & \multirow{4}{*}{$\begin{array}{l}\text { Wiener } \\
\text { filter }\end{array}$} & 14.312 & 2408.7 \\
\hline & $\begin{array}{l}\text { Gaussian } \\
\text { noise }\end{array}$ & & 16.764 & 1369.6 \\
\hline & Poisson noise & & 16.934 & 1317.1 \\
\hline & Speckle noise & & 16.787 & 1362.5 \\
\hline & $\begin{array}{c}\text { Salt \& Pepper } \\
\text { Noise }\end{array}$ & \multirow{4}{*}{$\begin{array}{c}\text { GMM } \\
\text { based } \\
\text { wavelet } \\
\text { Filter }\end{array}$} & 20.837 & 536.19 \\
\hline & $\begin{array}{l}\text { Gaussian } \\
\text { noise }\end{array}$ & & 26.835 & 134.74 \\
\hline & Poisson noise & & 22.677 & 351 \\
\hline & Speckle noise & & 22.537 & 362.48 \\
\hline
\end{tabular}

In the table I, we compare the filters based on performance parameter PSNR and MSE, which shows image clarity. If the restored image have greater PSNR and lower, value of MSE that's mean image is clearer.

By observing the output table, it found out that when the medical images are mixing with Poisson noise then median filter could be use. If medical images disturbed by salt \& pepper noise then median filter is best, if medical image is with Gaussian noise then GMM based wavelet filter can be used, if medical images getting mixed with speckle noise wiener filter is best.

when the satellite images is mixed with Poisson noise then median filter can be used, if satellite images mixed with salt \& pepper noise then median filter is best, if satellite image is with Gaussian noise then mean filter can be used, if satellite images getting mixed with speckle noise mean filter is usable.

when the natural images is blurred due to Poisson noise then median filter can be used, if natural images disturbed by salt \& pepper noise then median filter is best, if natural image is with Gaussian noise then GMM based wavelet filter can be used, if natural images getting mixed with speckle noise mean filter can apply.

when the texture images is mixed with Poisson noise then median filter can be used, if texture images disturbed by salt \& pepper noise then median filter is best, if texture image is with Gaussian noise then mean filter can be used, if texture images getting mixed with speckle noise mean filter is used.

\section{CONCLUSION}

In this paper present the method of segmentation algorithm for images and video based on the Gaussian mixture model. It segmented the image that means it gives the texture information of the image. In this paper we have used four different filters namely mean, median, wiener and wavelet filters based on Gaussian mixture model at restoration stage We have used four different types of noises namely salt \& pepper, Gaussian noise, Poisson noise and speckle noise. These noises added to the different diverse field of images like medical images, natural images, satellite images and texture images and that noisy images are filtered out by using each filter and we concluded that which filter can remove which type of noise smartly. So that, we can increases the system compatibility with different environmental conditions.

\section{ACKNOWLEDGMENT}

With deep sense of gratitude, I express my sincere thanks to my guide Prof. Anil Wanare in the Electronics and telecommunication department for his effective supervision. I thanks to all people who support me to carry this work.

\section{REFERENCES}

[1] Taeg Sang Cho, 2011 "Image restoration by matching gradient distribution": IEEE

[2] Christophoros Nikou "A Bayesian Framework for Image Segmentation with Spatially Varying Mixtures” IEEE

[3] Preethi K. 2011 "Denoising of surveillance video using adaptive Gaussian mixture Model based segmentation towards effective parameters measurement." : IJETT.

[4] Massimo Pi ardi, 2004 "Background subtraction techniques-review",International Conference on Systems, man and cybernetics, ICSMC

[5] H C Sateesh Kumar 1, K B Raja2, Venugopal K R2 and L M Patnaik3. "Automatic Image Segmentation using Wavelets.”: IJCSNS

[6] Anil L Wanare, Pratik D Shah and Dilip D Shah, January 2013, "Performance Analysis and Optimization of Linear restoration in Spatial Domain".. International Journal of Computer Applications 61(10):1-5.

[7] Matthew Marsh "A Literature Review of Image Segmentation Techniques and Matting for the Purpose of Implementing "Grab-Cut". 
[8] Mohamed Ali Mahjoub, 2009, "Image segmentation byadaptive distance based on EM algorithm": IJACSA.

[9] Mohand Saïd Allili, April 2012 "Wavelet Modeling Using Finite Mixtures of Generalized Gaussian Distributions: Application to Texture Discrimination and Retrieval": IEEE transaction, Vol. 21, No. 4.

[10] D. Brownrigg, Mar. 1984 "The weighted median filter," Commun. Assoc. Computer, pp. 807-818.

[11] S.-J. Ko and S.-J. Lee, Sept.1991., "Center weighted median filters and their applications to image enhancement," IEEE Trans. Circuits Syst., vol. 15, pp. 984-993.

[12] T. Sun and Y. Neuvo, 1994 "Detail-preserving median based filters in image processing," Pattern Recognit. Lett., vol. 15, pp. 341-347.

[13] D. Florencio and R. Schafer, Sept. 1994 "Decision-based median filter using local signal statistics," in Proc. SPIE Int. Symp, Visual Communications Image Processing, Chicago.

[14] How-Lung Eng, , and Kai-Kuang Ma, "Noise Adaptive Soft-Switching Median Filter'IEEE Transactions Vol. 10, No. 2, February 2001

[15] R. Yang, M. Gabbouj, and Y. Neuvo, "An efficient design method for optimal weighted median filtering," in
IEEE Int. Symp. Circuits Systems (ISCAS'94), Chicago, IL, Sept. 1994.

[16] Behrooz Ghandeharian, Hadi Sadoghi Yazdi And Faranak Homayouni "Modified Adaptive Center Eighted Median Filter For Uppressingimpulsive Noise In Images" in International Journal of Research and Reviews in Applied Sciences Volume1, Issue'3 (December-2009)

[17] S. Grace Chang,, SEPTEMBER 2000 "Adaptive Wavelet Thresholding for Image Denoising and Compression" IEEE Transactions On Image Processing, Vol. 9, No. 9.

[18] Mr. R. K. Sarawale1, Dr. Mrs. S.R. Chougule, June 2013 "Image Denoising using Dual-Tree Complex DWT and Double-Density Dual-Tree Complex DWT" International Journal of Advanced Research in Computer Engineering \& Technology (IJARCET) Volume 2, Issue 6 ,

[19] Taeg Sang Cho , awrence Zitnick ,Neel Joshi, 2011 "Image restoration by matching gradient distributions", IEEE Transactions on Pattern Analysis And Machine Intelligence.

[20] Xudong Jiang, April 2012 "Iterative Truncated Arithmetic Mean Filter and Its Properties", IEEE Transactions On Image Processing, Vol. 21, No. 4 